\title{
Research
}

\section{Association between National Early Warning Scores in primary care and clinical outcomes:}

\author{
an observational study in UK primary and secondary care
}

\begin{abstract}
Background

NHS England has mandated use of the

National Early Warning Score (NEWS), more recently NEWS2, in acute settings, and suggested its use in primary care. However, there is reluctance from GPs to adopt NEWS/ NEWS2.
\end{abstract}

\section{Aim}

To assess whether NEWS calculated at the point of GP referral into hospital is associated with outcomes in secondary care.

\section{Design and setting}

An observational study using routinely collected data from primary and secondary care.

\section{Method}

NEWS values were prospectively collected for 13047 GP referrals into acute care between July 2017 and December 2018. NEWS values were examined and multivariate linear and logistic regression used to assess associations with process measures and clinical outcomes.

\section{Results}

Higher NEWS values were associated with faster conveyance for patients travelling by ambulance, for example, median 94 minutes (interquartile range [IQR] 69-139) for NEWS 27; median 132 minutes, (IQR 84-236) for NEWS $=0$ to 2): faster time from hospital arrival to medical review (54 minutes [IQR 25-114] for NEWS $\geq 7$; 78 minutes [IQR 34-158] for NEWS = 0 to 2); as well as increased length of stay (5 days [IQR 2-11] versus 1 day [IQR $0-5])$; intensive care unit admissions $(2.0 \%$ versus $0.5 \%$ ); sepsis diagnosis $(11.7 \%$ versus $2.5 \%)$; and mortality, for example, 30-day mortality $12.0 \%$ versus $4.1 \%$ for NEWS $\geq 7$ versus NEWS $=0$ to 2 , respectively. On average, for patients referred without a NEWS value (NEWS = NR), most clinical outcomes were comparable with patients with NEWS $=3$ to 4 but ambulance conveyance time and time to medical review were comparable with patients with NEWS $=0$ to 2

\section{Conclusion}

This study has demonstrated that higher NEWS values calculated at GP referral into hospital are associated with a faster medical review and poorer clinical outcomes.

\section{Keywords}

continuity of care; general practice; national early warning score; patient safety; primary care; routinely collected data; secondary care.

\section{INTRODUCTION}

Early warning scores (EWS) are recommended in UK secondary care ${ }^{1}$ to aid detection of deterioration and improve patient outcomes. Scores are calculated from physiological observations, with higher scores indicating patients were more unwell. Though developed for hospitals, studies of EWS in pre-hospital settings have been shown to predict outcomes such as hospital stay and mortality; ${ }^{2-6}$ however, those studies were mostly in ambulance and non-UK settings.

To align scoring across the country, the Royal College of Physicians (RCP) developed the National Early Warning Score (NEWS) for secondary care in 2012.7 NEWS comprises respiratory rate oxygen saturation, temperature, systolic blood pressure, heart rate, and level of consciousness. Each is scored $0-3$ and combined to give an overall score with two additional points for supplemental oxygen. Scores lie from 0 to 20, with higher scores resulting from abnormal physiological measurements (Supplementary Figure S1). NEWS has been demonstrated to be a good sepsis screening tool ${ }^{8}$ and its structured observations are recommended nationally to aid recognition of sepsis..$^{8-1}$

In 2015, the West of England Academic Health Sciences Network introduced NEWS,

LJ Scott, MSc, senior research associate in quantitative research and evidence synthesis: NM Redmond, PhD, research fellow, Nationa Institute for Health Research Applied Research Collaboration West (NIHR ARC West), University Hospitals Bristol NHS Foundation Trust, Bristol; Population Health Sciences, Bristol Medica School, University of Bristol, Bristol. A Tavare MSc, FRCGP, primary care clinical lead; $\mathbf{H}$ Little BA, MSc, patient safety improvement lead and senior project manager, West of England Academic Health Science Network, Bristol. S Srivastava, FRCP, consultant physician and associate medical director, North Bristol NHS

Trust, Bristol. A Pullyblank, MD, FRCS, consultan surgeon, North Bristol NHS Trust, and medical director, West of England Academic Health via regional training programmes, into all healthcare settings across the region within five clinical commissioning groups, ${ }^{12,13}$ including six acute trusts, two mental health trusts, six community providers, the South West Ambulance Service, primary care practices, and GP out-of-hours services. As per the RCP's escalation criteria, ${ }^{7}$ NEWS $\geq 5$ was recommended for referral into acute care if clinically appropriate.

NEWS2, an update of NEWS (Supplementary Figure S2), is now mandated by NHS England for acute hospitals and recommended in pre-hospital settings including primary care. ${ }^{7,9-11,14,15}$ An advantage of calculating NEWS/NEWS2 while patients are in primary care is to start the track-and-trigger process earlier. However, there is reluctance from GPs to adopt NEWS/NEWS2 for a number of reasons, such as a preference for observing symptoms, clinical instinct, and a lack of validated studies conducted in primary care; GPs also report time restraints restricting the appropriateness of a full set of observations. ${ }^{16,17}$ There is currently little evidence about how NEWS in these settings influences patient outcomes, ${ }^{4}$ though some is beginning to emerge. ${ }^{2-5,13,16,18}$

The objective of this study was to investigate whether NEWS calculated at the point of GP

Science Network, Bristol; North Bristol NHS Trust, Bristol.

\section{Address for correspondence}

Lauren J Scott, National Institute for Health Research Applied Research Collaboration West 9th Floor, Whitefriars, Lewins Mead, Bristol BS1 2NT, UK

Email: Lauren.Scottabristol.ac.uk

Submitted: 7 October 2019; Editor's response: 8 November 2019; final acceptance: 7 January 2020.

\section{(c)The Authors}

This is the full-length article lpublished online 7 Apr 2020) of an abridged version published in print. Cite this version as: $\mathbf{B r} \mathbf{J}$ Gen Pract 2020; DOI: https://doi.org/10.3399/bjgp20X709337 


\section{How this fits in}

The National Early Warning Score (NEWS) is mandated for use in secondary care and recommended for use in out-of-hospital settings. In the West of England, NEWS is used across the whole system including primary care; however, there is reluctance from GPs, both locally and nationally, to adopt NEWS. This study demonstrates that NEWS values recorded at the point of referral into hospital are associated with poorer clinical outcomes and can be used to improve the process of care and prioritise the sickest patients.

referral to hospital affects timely process of care, that is, sicker patients seen faster, and to investigate clinical outcomes in hospital.

\section{METHOD}

\section{Design and setting}

This was an observational study using routinely collected data from primary and secondary care.

\section{Data acquisition}

One trust in the region, covering a population of approximately 500000 people and the largest hospital in the South West, ${ }^{19}$ uses the Clinical Information Systems Suite (CISS) database to record all acute admissions into the medical admissions unit (MAU). Time of referral, time of arrival in MAU, time of doctor review, and reviewing doctors' grade are prospectively recorded on the database, along with symptoms on referral. In June 2017, a 'NEWS on referral' field was added to this database; from this time GPs were
Figure 1. Time from GP referral to arrival in hospital by NEWS on referral and ambulance conveyance. $\mathrm{Cl}=$ confidence interval. GMR = geometric mean ratio. NEWS = National Early Warning Score. $N R=$ not recorded. asked for component observations of NEWS and a score was calculated and recorded. GPs aware of NEWS may have calculated a score themselves before deciding to refer, but others simply provided component parts because they were requested to when an admission was arranged. Data from July 2017 to December 2018 inclusive were included in this analysis.

\section{Data linkage}

Information analysts at the trust used NHS numbers to link the CISS data to local secondary use service data, to identify length of hospital stay (LOS), primary diagnosis, intensive care unit (ICU) admission, and mortality for each patient. These linked data were anonymised and sent to the project team for analysis.

\section{Outcomes}

The authors examined whether NEWS was associated with the following process measures and clinical outcomes: time from referral to arrival in hospital (by ambulance or other transport), time from arrival to review by doctor, grade of reviewing doctor, LOS, suspicion of sepsis (SOS), primary diagnosis of sepsis, ICU admission, 2-day mortality, and 30-day mortality.

\section{Data preparation and derivations}

The authors were interested in medical admissions from GPs to MAU, including patients seen in ambulatory care. Surgical patients and those referred from the emergency department (ED) or other hospital locations were excluded. Repeated data, that is, where patient ID and referral date appeared more than once $(<1 \%)$, were excluded due to uncertainty over which data were accurate.

NEWS values were grouped into five categories: 0 to 2,3 to 4,5 to $6, \geq 7$, and not recorded (NR). These categories were chosen in line with secondary care escalation trigger scores of 3,5, and 7.14 Patients who had some component observations of NEWS recorded but no NEWS value calculated and recorded in the database were grouped in NEWS $=$ NR.

Suspicion of sepsis was derived from the ICD-10 primary diagnosis codes using a reference list of approximately 250 codes relating to bacterial infections. ${ }^{20,21}$ Primary diagnosis of sepsis was a subset comprising only ICD-10 codes A40 ('streptococcal sepsis') and A41 ("other sepsis').

An 'ambulance conveyance' variable was derived from a free-text field containing GP-reported details of presenting complaints: Ambulance $=$ Yes if the word 


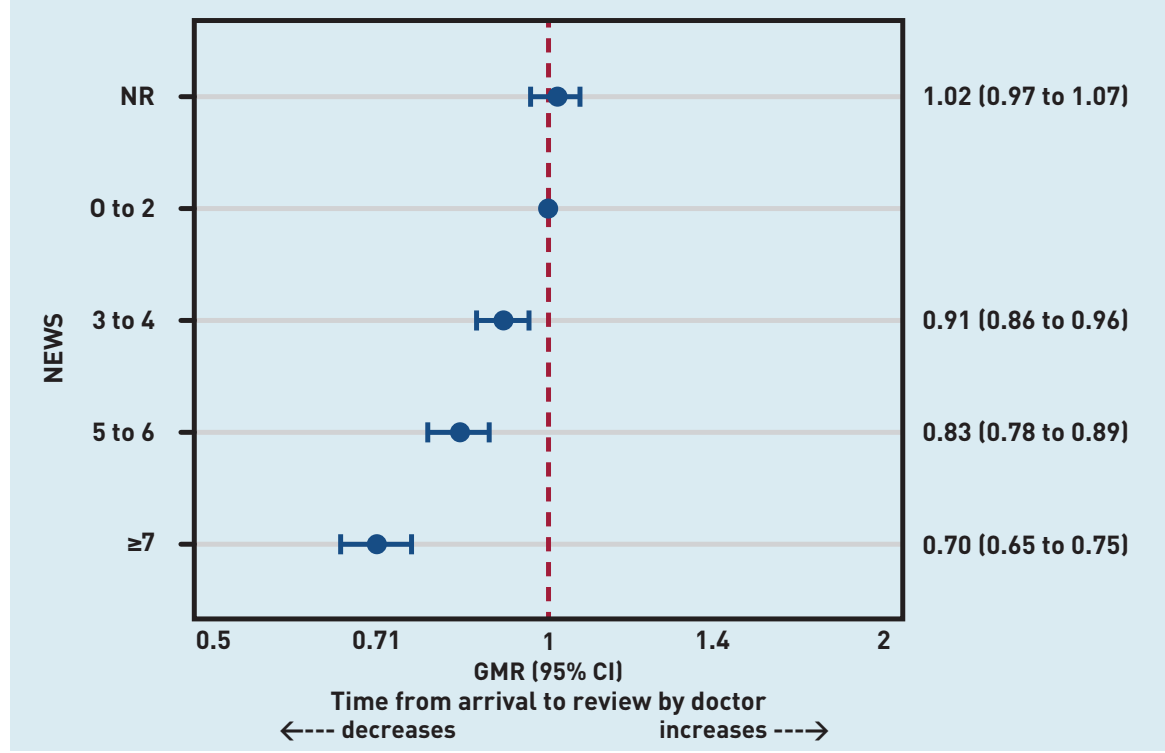

Figure 2. Time from hospital arrival to doctors' review by NEWS on referral. $\mathrm{Cl}=$ confidence interval. $G M R=$ geometric mean ratio. NEWS = National Early Warning Score. $N R=$ not recorded 'ambulance' was mentioned but not preceded by the word 'no' (that is, not 'no ambulance'); otherwise Ambulance = No (this variable was set to missing if no presenting complaints were recorded).
Time from referral to arrival in MAU and arrival to medical review were calculated from the times recorded in the CISS database. Both date and time were recorded for referral, but only time was recorded for arrival and review. Therefore, if care crossed midnight, the difference between the two appeared negative, for example, if referral time was $10.00 \mathrm{pm}$ and arrival time was $1.00 \mathrm{am}$, calculated time would be -21 hours. To account for this, times calculated between -24 and -1 hours, were assumed to cross midnight and 24 hours were added; times calculated between -1 and $<0$ hours were assumed to be an error, where times were swapped around during data entry and were multiplied by -1 . Times recorded as 00:00 appeared far more than other times; it was assumed this time was entered if true time was unknown and therefore set to missing.

\section{Statistical analysis}

Continuous and categorical data were summarised using medians and interquartile ranges (IQR), and counts and percentages, respectively. To investigate the

\section{Table 1. Summary of outcomes by National Early Warning Scores (NEWS) on referral}

\begin{tabular}{|c|c|c|c|c|c|c|c|c|c|c|c|c|}
\hline \multirow{2}{*}{$\begin{array}{l}\text { Process outcomes } \\
\text { Time from referral to } \\
\text { arrival in hospital, } \\
\text { median minutes (IQR) })^{a, b}\end{array}$} & \multicolumn{2}{|c|}{$\begin{array}{c}\text { NEWS }=\text { NR } \\
(N=2848)\end{array}$} & \multicolumn{2}{|c|}{$\begin{array}{c}\text { NEWS }=0 \text { to } 2 \\
(N=5514)\end{array}$} & \multicolumn{2}{|c|}{$\begin{array}{c}\text { NEWS }=3 \text { to } 4 \\
(N=2162)\end{array}$} & \multicolumn{2}{|c|}{$\begin{array}{c}\text { NEWS }=5 \text { to } 6 \\
(N=1458)\end{array}$} & \multicolumn{2}{|c|}{$\begin{array}{l}N E W S \geq 7 \\
(N=1065)\end{array}$} & \multicolumn{2}{|c|}{$\begin{array}{c}\text { Overall } \\
(N=13047)\end{array}$} \\
\hline & 79 & $(48-142)$ & 74 & $(47-122)$ & 79 & $(50-126)$ & 85 & (57-132) & 90 & $(64-127)$ & 79 & $(50-129)$ \\
\hline Conveyed by ambulance & 133 & (82-240) & 132 & $(84-236)$ & 115 & (78-193) & 104 & (77-159) & 94 & (69-139) & 116 & (78-200) \\
\hline Conveyed by other transport ${ }^{c}$ & 71 & $(44-124)$ & 68 & $(44-106)$ & 72 & $(46-110)$ & 75 & (49-118) & 85 & (60-119) & 71 & $(46-112)$ \\
\hline $\begin{array}{l}\text { Time from arrival in hospital } \\
\text { to review by doctor, median } \\
\text { minutes (IQR) }\end{array}$ & 80 & (36-156) & 78 & (34-158) & 72 & $(34-144)$ & 68 & (31-130) & 54 & $(25-114)$ & 74 & (33-148) \\
\hline \multicolumn{13}{|l|}{ Grade of reviewing doctor, $n(\%)$ e } \\
\hline Foundation year 1 & 172 & $(6.4)$ & 357 & (6.8) & 148 & (7.1) & 113 & (8.1) & 68 & (6.5) & 858 & (6.9) \\
\hline Foundation year $2 /$ senior house officer & 1387 & $(51.8)$ & 2479 & $(47.4)$ & 1208 & (58.0) & 908 & $(64.9)$ & 692 & $(66.6)$ & 6674 & (53.7) \\
\hline Specialty registrar & 647 & $(24.2)$ & 1036 & (19.8) & 443 & (21.3) & 317 & $(22.7)$ & 264 & $(25.4)$ & 2707 & (21.8) \\
\hline Consultant & 78 & (2.9) & 145 & (2.8) & 38 & (1.8) & 20 & $(1.4)$ & 11 & (1.1) & 292 & $(2.4)$ \\
\hline $\mathrm{GP}^{\mathrm{f}}$ & 384 & (14.3) & 1177 & $(22.5)$ & 242 & $(11.6)$ & 39 & (2.8) & 4 & $(0.4)$ & 1846 & $(14.9)$ \\
\hline \multicolumn{13}{|l|}{ Clinical outcomes } \\
\hline Length of stay, median days $(I Q R)^{g}$ & 2 & $(0-6)$ & 1 & $(0-5)$ & 3 & (1-7) & 4 & $(2-10)$ & 5 & $(2-11)$ & 2 & $(0-7)$ \\
\hline Admission to ICU, $n(\%)^{g}$ & 17 & (0.7) & 24 & (0.5) & 11 & (0.5) & 15 & (1.1) & 20 & (2.0) & 87 & $(0.7)$ \\
\hline Suspicion of sepsis, $n(\%)^{\text {h }}$ & 374 & (17.9) & 764 & (19.8) & 655 & (36.9) & 676 & (51.3) & 669 & (67.1) & 3138 & (31.3) \\
\hline Primary diagnosis of sepsis, $\mathrm{n}(\%)^{\mathrm{h}}$ & 54 & $(2.6)$ & 97 & $(2.5)$ & 90 & (5.1) & 102 & (7.7) & 117 & (11.7) & 460 & $(4.6)$ \\
\hline 2-day mortality, $n(\%)^{\mathrm{h}}$ & 13 & $(0.6)$ & 12 & (0.3) & 12 & $(0.7)$ & 10 & (0.8) & 24 & $(2.4)$ & 71 & (0.7) \\
\hline 30-day mortality, $n(\%)^{\text {h }}$ & 147 & (7.0) & 158 & (4.1) & 122 & (6.9) & 117 & (8.9) & 120 & (12.0) & 664 & $(6.6)$ \\
\hline \multicolumn{13}{|c|}{ 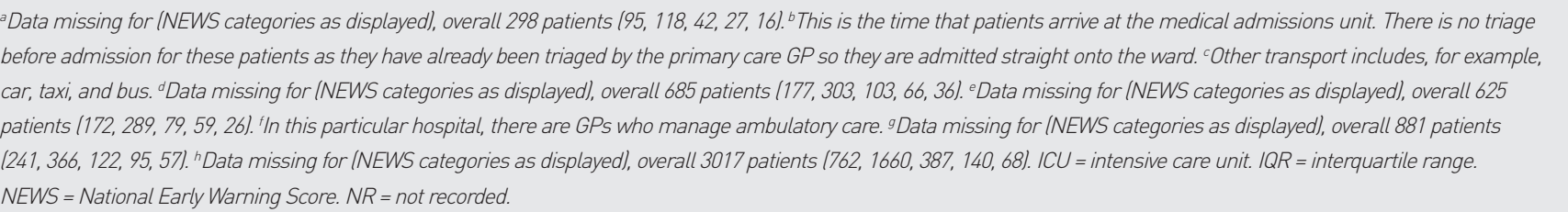 } \\
\hline
\end{tabular}




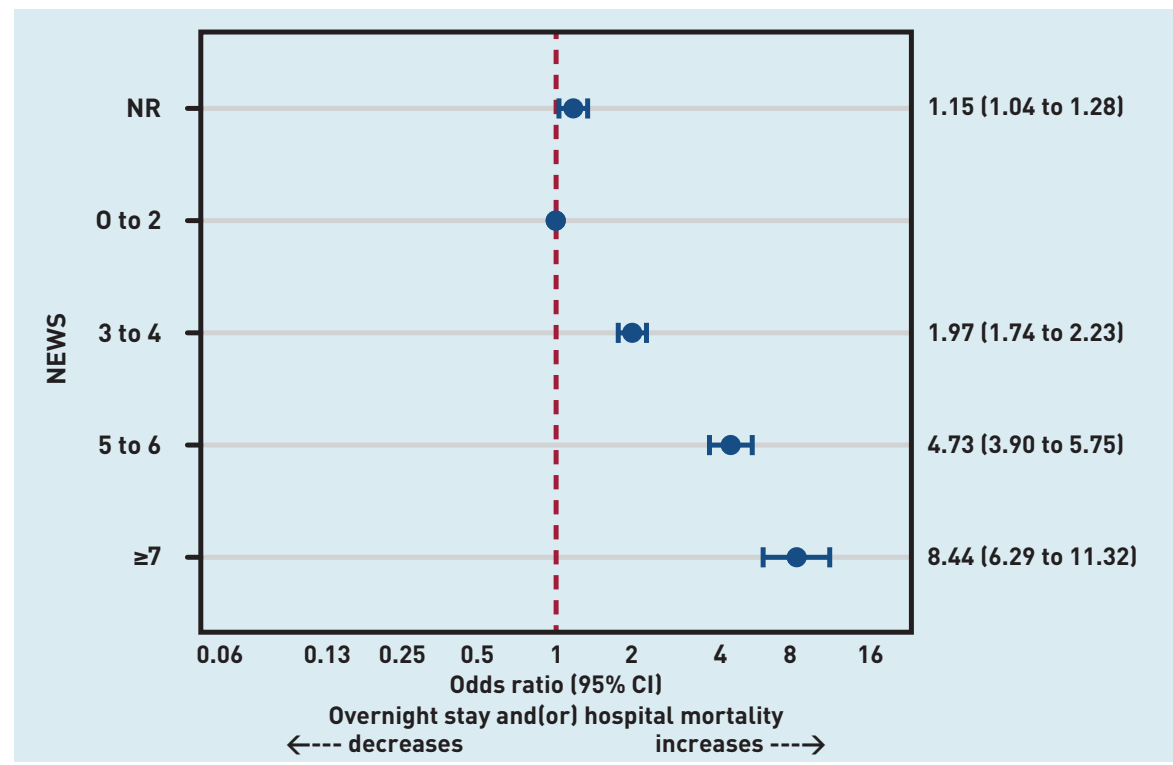

Figure 3. Overnight hospital stay and/or hospital mortality by NEWS on referral. $C l=$ confidence interval. NEWS $=$ National Early Warning Score. $N R=$ not recorded. effects of referral NEWS, binary outcomes were analysed using multivariate logistic regression and presented as odds ratios (ORs) with corresponding 95\% confidence intervals (Cls). Continuous outcomes were analysed using multivariate linear regression; due to poor model fit, continuous outcomes were log-transformed lwith zero values replaced with 0.5 before transformation), and results presented as geometric mean ratios (GMRs) with 95\% Cls. As the model fit for LOS was still poor following log-transformation, it was dichotomised (no overnight stay versus overnight stay and/or hospital mortalityl and analysed using logistic regression as above. All models were adjusted for age and sex. Model fit was tested using standard methods and outliers were excluded. The analysis for referral to arrival time by conveyance method was calculated by adding a NEWS by conveyance method interaction term. Missing data are described but all analyses included complete cases; no imputation was performed. Stata 15 (version 1) was used for all data checking, cleaning, and analyses.

\section{RESULTS}

\section{Patient demographics}

There were 13047 medical admissions from GPs to MAU between 01 July 2017 and 31 December 2018. The median age of patients was 69 years (IQR 51-82) and 7285/13 047 $(56 \%)$ were female. The percentage who had NEWS recorded was reasonably stable across the time period: $74 \%$ in July 2017 and $78 \%$ in December 2018. Overall, $42 \%$, $17 \%, 11 \%, 8 \%$, and $22 \%$ of patients had NEWS $=0$ to $2, N E W S=3$ to $4, N E W S=5$ to
6, NEWS $\geq 7$, and NEWS $=$ NR, respectively. Of those with NEWS recorded, the median score was 2 (IQR 0-4).

On average, NEWS was higher in older patients; 523/4760 (11\%) patients aged 16 to 59 years had NEWS $\geq 5$ compared with $2000 / 8287(24 \%)$ patients aged $\geq 60$ years. The percentage of patients with NEWS = NR was the same in the two age groups (22\%). NEWS values were less likely to be recorded at 'night' $(8.00 \mathrm{pm}$ to $8.00 \mathrm{am})$ than during the 'morning' ( $8.00 \mathrm{am}$ to $2.00 \mathrm{pm}$ ) or 'afternoon' (2.00 pm to $8.00 \mathrm{pm}$ ): 845/1273 (66.4\%), 4754/5871 (81.0\%), and 4590/5869 (78.2\%), respectively.

\section{Referral to arrival in hospital}

Median time from GP referral to arrival in MAU was 79 minutes (IQR 50-129, Table 1). For patients conveyed by ambulance (21\%), as NEWS increased, referral to arrival time decreased, and patients with NEWS $=$ NR had very similar times to those with NEWS $=0$ to 2 (Figure 1, Table 1). The relationship was unclear for patients conveyed by other transport, for example, car, taxi, or bus (Figure 1).

On average, patients conveyed by ambulance had higher NEWS values (34\% versus $15 \%$ NEWS $\geq 5$ ) and slower conveyances 1116 minutes versus 71 minutes) than patients using other transport.

\section{Arrival in hospital to review by doctor}

Median time from arrival to review was 74 minutes (IQR 33-148) (Table 1). As NEWS value increased, average arrival to review time decreased; for patients with NEWS $=N R$, times were similar to patients with NEWS $=0$ to 2 (Figure 2, Table 1). Patients with higher NEWS were more likely to be seen by a senior doctor (specialty registrar or consultant) than patients with lower NEWS (Table 1); in particular, patients with NEWS $\geq 7$ were more likely than NEWS $=0$ to 2 (OR $1.30,95 \% \mathrm{Cl}=1.11$ to 1.51 (Supplementary Figure S3).

\section{Length of stay}

As NEWS increased, average LOS increased, with a median of 1 day (IQR 0-5), 3 days (IQR 1-7), 4 days (IQR 2-10), and 5 days (IQR 2-11) for patients with referral NEWS of 0 to 2,3 to 4,5 to 6 , and $\geq 7$, respectively (Table 1). Patients with NEWS $=$ NR had a median LOS of 2 days (IQR $0-6$ ). The analysis of overnight stay and/or hospital mortality reflects this relationship, for example, a patient with NEWS $\geq 7$ had $>8$ times the odds compared with someone 


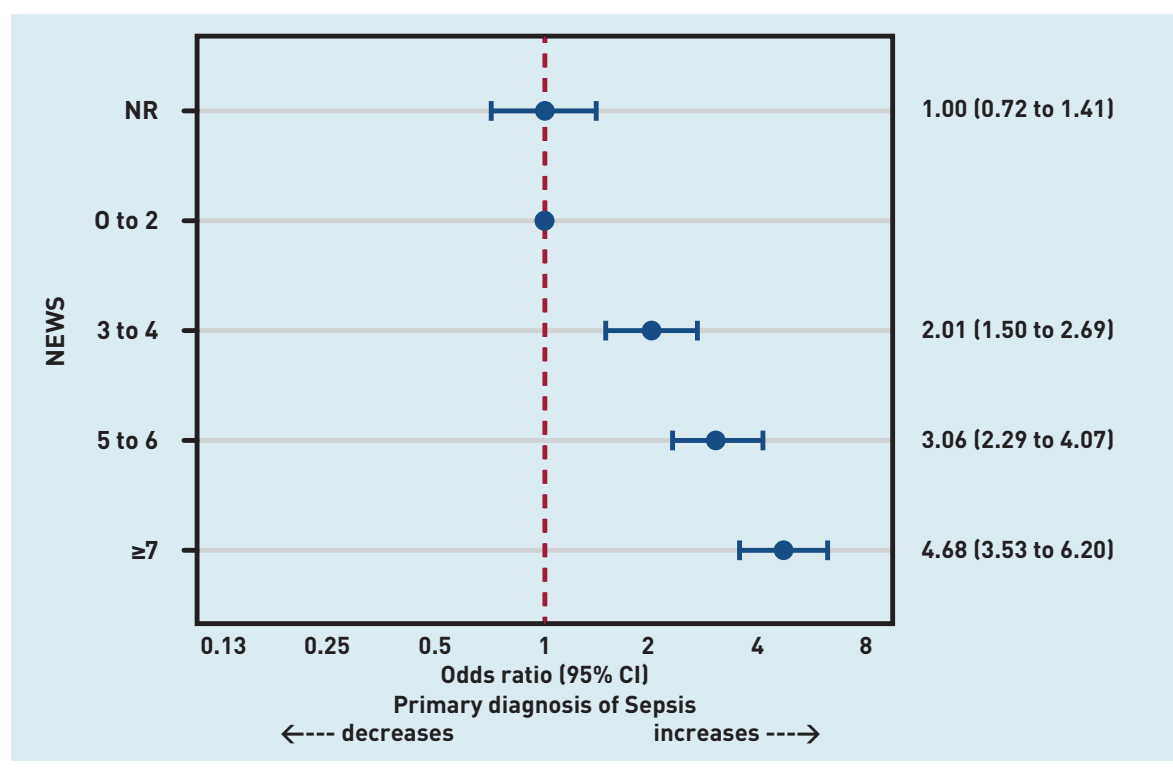

Figure 4. Primary diagnosis of sepsis by NEWS on referral. $\mathrm{Cl}=$ confidence interval. NEWS = National Early Warning Score. $N R=$ not recorded.

Figure 5. Two-day mortality by NEWS on referral. $\mathrm{Cl}=$ confidence interval. NEWS = National Early Warning Score. $N R=$ not recorded . with NEWS $=0$ to 2 lOR $8.44,95 \% \mathrm{Cl}=6.29$ to 11.32) (Figure 3).

\section{ICU admission}

Overall, only 87/12 $166(0.7 \%)$ patients were admitted to ICU. The odds of ICU admission and/or hospital mortality increased as NEWS increased, for example, NEWS $\geq 7$ compared with NEWS $=0$ to 2 , OR $3.08,95 \% \mathrm{Cl}=2.36$ to 4.00; odds for patients with NEWS $=$ NR were higher than NEWS $=0$ to 2 (Table 1 , Supplementary Figure S4).

\section{Sepsis}

A total of $460 / 10030$ (4.6\%) patients had a primary diagnosis of sepsis and $3138 / 10030$ (31.3\%) had SOS (Table 1). The odds of sepsis and SOS increased as

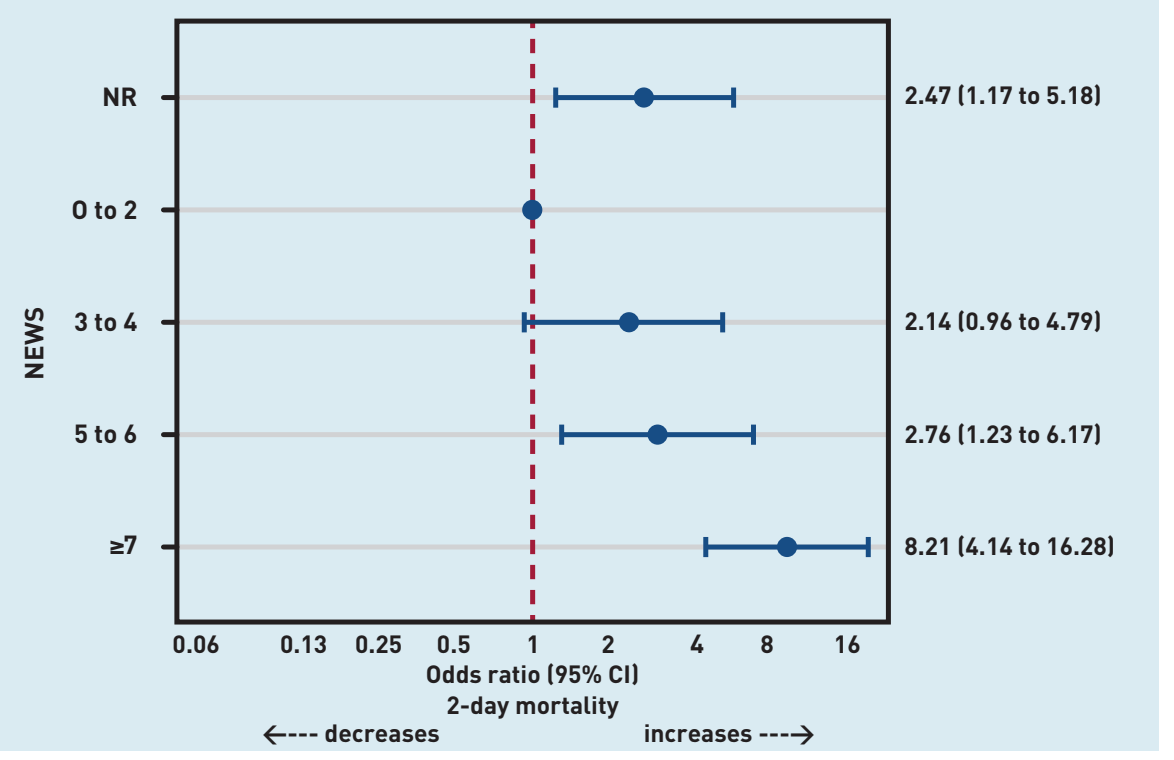

NEWS on referral increased; for patients without NEWS, the odds of sepsis and SOS were the same as, and slightly lower than, NEWS $=0$ to 2, respectively (Figure 4 and Supplementary Figure S5).

\section{Mortality}

A total of $71 / 10030(0.7 \%)$ and $664 / 10030$ (6.6\%) patients died within 2 days and 30 days of admission, respectively (Table 1). As NEWS increased, 2-day mortality increased, for example, for NEWS $\geq 7$ compared with NEWS $=0$ to 2, OR $8.21,95 \% \mathrm{Cl}=4.14$ to 16.28 (Figure 5). The odds for patients with NEWS $=$ NR were higher than NEWS $=0$ to 2 (OR 2.47, $95 \% \mathrm{Cl}=1.17$ to 5.18 ). A similar pattern was seen for 30-day mortality (Supplementary Figure S6).

\section{DISCUSSION}

\section{Summary}

This is the first UK study investigating the association between NEWS at the time of referral from primary care and resulting process measures and clinical outcomes in secondary care. Importantly, this study found higher NEWS values were associated with increased LOS, ICU admissions, sepsis (suspected and diagnosed), mortality (2-day and 30-day), decreased time from referral to arrival for patients conveyed by ambulance, and decreased time from arrival in hospital to doctor review.

Patients with NEWS = NR had increased LOS, ICU admissions, and mortality compared with patients with NEWS $=0$ to 2 but conveyances and time to treatment on average as long as, or even longer than, patients with NEWS $=0$ to 2 . This suggests the group without NEWS may include a spectrum of sick and less sick patients, and highlights a potentially missed opportunity for earlier conveyance and review of sicker patients, which may have been mitigated if NEWS had been calculated. Alternatively, this group may include patients with clear referral pathways, for example, patients who have had a stroke, for whom NEWS would not have provided additional information. However, primary diagnosis data do not support this hypothesis, for example, $16 / 2848(0.56 \%)$ were myocardial infarctions and $12 / 2848(0.42 \%)$ were strokes.

Unlike the other clinical outcomes, patients with NEWS = NR had the same odds of diagnosis of sepsis and slightly lower odds of SOS than patients with NEWS $=0$ to 2. As NEWS is recommended by the National Institute for Health and Care Excellence to identify sepsis, ${ }^{9}$ GPs might be more inclined to calculate NEWS, or its component parts, if they suspect sepsis. 


\section{Strengths and limitations}

The strengths of this analysis are threefold: first, the dataset is large (>13000 referrals) with relatively little missing data. Second, data used are 'real-world' prospective data from a busy UK NHS trust, representative of other regional UK areas. Third, owing to the databases used, the authors had the ability to link patients' outcomes robustly, which is often not possible.

There are some potential limitations. Although the dataset is large, it is based on one hospital so may not be generalisable. As this is routine, retrospective NHS data, it is likely to contain data entry errors, even though methodically cleaned before analysis. Further, it may not reflect the full context of the interaction between primary and secondary care, for example, if the secondary care staff member(s) receiving patient referrals is unavailable when a patient arrives, they may be seen in ED first and therefore would not appear in this dataset. This study found that patients with higher NEWS were reviewed by a doctor faster than those with lower scores, so it is possible that the observed relationship between high NEWS values and poorer clinical outcomes could be stronger than shown in this analysis, but was moderated by faster review and treatment. Also, it is not known whether decreased ambulance conveyance times and arrival to review times with higher NEWS were due to NEWS itself or because healthcare staff recognised that patients were sicker. There may be missing data from the ambulance conveyance status due to its free-text origins, and so these data may not be robust. Finally, this study's data pertain to NEWS rather than the updated NEWS2. The key changes for NEWS2 are a new oxygen saturation section for patients with hypercapnic respiratory failure and the consideration of new-onset confusion alongside level of consciousness..$^{14}$ Although these changes would affect NEWS values in a subset of patients, the authors do not believe these changes would significantly alter the present findings.

\section{Comparison with existing literature}

The results presented in this study are in line with data from secondary care, ${ }^{22-26}$ where patient mortality has been shown to be related to NEWS values. In a recent systematic review of EWS in pre-hospital settings, ${ }^{4}$ ambulance service data indicated EWS had good accuracy for prediction of death within 48 hours. In addition, patients with $\mathrm{EWS}=0$ were very unlikely to deteriorate and higher scores resulted in higher risk of deterioration. The present data agree with these findings in terms of mortality, sepsis, LOS, and ICU admission.

A recent study of patients transported by ambulance to hospital in Finland ${ }^{18}$ found a 1-day mortality rate of $0.21 \%$ for NEWS $=0$ to 4 and $21.3 \%$ for NEWS $\geq 7$ calculated retrospectively from clinical parameters recorded by the ambulance crew. They also found a 30-day mortality rate of $2.1 \%$ for NEWS $=0$ to 4 and $33.1 \%$ for NEWS $\geq 7$. These figures differ from the present findings, particularly for NEWS $\geq 7$, most likely because of their inclusion of patients with trauma. Other studies $3,5,13,27$ that investigated the diagnostic accuracy of NEWS/NEWS2 in pre-hospital or ED settings to predict mortality all found that, as NEWS increased, mortality increased, in line with the findings presented in this article. One ED study also found that, as NEWS increased, sepsis increased $10.4 \%$ for NEWS $=0$ to 2 and $17.5 \%$ for NEWS $\geq 7$ ). ${ }^{27}$ These results are lower than the present findings for low NEWS and higher for high NEWS; this is likely because of the patient cohort undergoing GP 'triage' before inclusion and the exclusion of surgical patients in this study. It is difficult to directly compare the present results to any of these studies as they are based on retrospective calculations of NEWS, whereas NEWS was in use as a track-and-trigger system in the present patient population and therefore clinical actions triggered by these scores may have improved outcomes.

\section{Implications for practice}

The findings should go some way to increase GPs' trust in using NEWS (now NEWS2) to communicate patient acuity to ambulance and secondary care staff. It should reassure GPs both locally and nationally that, when used in conjunction with clinical judgement, calculating NEWS in primary care is worthwhile in terms of its impact on improving clinical processes of assessment and treatment of their patients.

Patients who came to hospital via their own transport arrived quicker than by ambulance. Unlike in ambulance conveyances, however, higher NEWS did not correlate with a faster transfer. This means that, while NEWS may be used by ambulance call handlers and paramedics for triage, patients and carers could benefit from education about NEWS to ensure that, if they have a higher score, this can be communicated, and they can ensure a prompt transfer to hospital. 


\section{REFERENCES}

1. National Institute for Health and Care Excellence. Acutely ill patients in hospital: recognition of and response to acute illness in adults in hospital. CG50. London: NICE, 2007. https://www.nice.org.uk/guidance/cg50/resources/ acutely-ill-adults-in-hospital-recognising-and-responding-to-deteriorationpdf-975500772037 (accessed 4 Mar 2020).

2. Lane DJ, Wunsch $H$, Saskin R, et al. Assessing severity of illness in patients transported to hospital by paramedics: external validation of 3 prognostic scores. Prehosp Emerg Care 2020; 24(2): 273-281.

3. Martin-Rodriguez F, Lopez-Izquierdo R, Del Pozo Vegas C, et al. A multicenter observational prospective cohort study of association of the prehospital national early warning score 2 and hospital triage with early mortality. Emerg Med Int 2019; 2019: 5147808

4. Patel R, Nugawela MD, Edwards HB, et al. Can early warning scores identify deteriorating patients in pre-hospital settings? A systematic review. Resuscitation 2018; 132: 101-111.

5. Pirneskoski J, Kuisma M, Olkkola KT, Nurmi J. Prehospital National Early Warning Score predicts early mortality. Acta Anaesthesiol Scand 2019; 63(5): 676-683.

6. Williams TA, Tohira H, Finn J, et al. The ability of early warning scores (EWS) to detect critical illness in the prehospital setting: a systematic review. Resuscitation 2016; 102: 35-43.

7. Royal College of Physicians. National Early Warning Score (NEWS) standardising the assessment of acute-illness severity in the NHS. London: RCP, 2012.

8. Goulden R, Hoyle MC, Monis J, et al. qSOFA, SIRS and NEWS for predicting inhospital mortality and ICU admission in emergency admissions treated as sepsis. Emerg Med J 2018; 35(6): 345-349.

9. National Institute for Health and Clinical Excellence. Sepsis: recognition, diagnosis and early management. NG51. NICE: London, 2017. https://www.nice. org.uk/guidance/ng51 (accessed 4 Mar 2020).

10. NHS England. Sepsis guidance implementation advice for adults. 2017. https://wnw.england.nhs.uk/wp-content/uploads/2017/09/sepsis-guidanceimplementation-advice-for-adults.pdf (accessed 4 Mar 2020).

11. National Confidential Enquiry into Patient Outcome and Death. Just Say Sepsis! A review of the process of care received by patients with sepsis. 2015. https:// wuw.ncepod.org.uk/2015report2/downloads/JustSaySepsis_FullReport.pdf laccessed 4 Mar 2020).

12. Institute for Healthcare Improvement. The Breakthrough Series: IHI's Collaborative Model for Achieving Breakthrough Improvement. Boston, MA: Institute for Healthcare Improvement, 2003. http://www.ihi.org/resources/ Pages/IHIWhitePapers/TheBreakthroughSeriesIHIsCollaborative ModelforAchievingBreakthroughlmprovement.aspx (accessed 4 Mar 2020).
13. Scott LJ, Redmond NM, Garrett J, et al. Distributions of the National Early Warning Score (NEWS) across a healthcare system following a large-scale rollout. Emerg Med J 2019; 36(5): 287-292.

14. Royal College of Physicians. National Early Warning Score (NEWS) 2: Standardising the assessment of acute-illness severity in the NHS. London: RCP, 2017. https://www.rcplondon.ac.uk/projects/outputs/national-earlywarning-score-news-2 (accessed 4 Mar 2020)

15. Royal College of Emergency Medicine. Position statement: National Early Warning Score (NEWS) for adult patients attending emergency departments. 2016. https://www.rcem.ac.uk/docs/News/CEM10125-Position\%20 statement $\% 20-\% 20$ NEWS $\% 20$ for\%20adult\%20patients\%20attending $\% 20$ EDs\%20-\%20June\%202016.pdf (accessed 4 Mar 2020).

16. Brangan E, Banks J, Brant H, et al. Using the National Early Warning Score (NEWS) outside acute hospital settings: a qualitative study of staff experiences in the West of England. BMJ Open 2018; 8(10): e022528.

17. Mahase E. GPs warn over scoring system for calling ambulances for patients. BMJ 2019; 367: 15814.

18. Hoikka M, Silfvast T, Ala-Kokko TI. Does the prehospital National Early Warning Score predict the short-term mortality of unselected emergency patients? Scand J Trauma Resusc Emerg Med 2018; 26(1): 48.

19. Indeed. Welcome North Bristol NHS Trust. 2020. https://mww.indeed.co.uk/ $\mathrm{cmp} /$ North-Bristol-Nhs-Trust/about laccessed 4 Mar 2020).

20. Imperial College Health Partners. Suspicion of sepsis insights dashboard. 2019. https://imperialcollegehealthpartners.com/project/suspicion-of-sepsisinsights-dashboard/ (accessed 4 Mar 2020).

21. Inada-Kim M, Page B, Maqsood I, Vincent C. Defining and measuring suspicion of sepsis: an analysis of routine data. BMJ Open 2017; 7(6): e014885.

22. Alam N, Hobbelink EL, van Tienhoven AJ, et al. The impact of the use of the Early Warning Score (EWS) on patient outcomes: a systematic review. Resuscitation 2014; 85(5): 587-594.

23. Gao H, McDonnell A, Harrison DA, et al. Systematic review and evaluation of physiological track and trigger warning systems for identifying at-risk patients on the ward. Intensive Care Med 2007; 33(4): 667-679.

24. McNeill G, Bryden D. Do either early warning systems or emergency response teams improve hospital patient survival? A systematic review. Resuscitation 2013; 84(12): 1652-1667.

25. Smith GB, Prytherch DR, Schmidt PE, Featherstone PI. Review and performance evaluation of aggregate weighted 'track and trigger' systems. Resuscitation 2008; 77(2): 170-179.

26. Smith ME, Chiovaro JC, O'Neil M, et al. Early warning system scores for clinical deterioration in hospitalized patients: a systematic review. Ann Am Thorac Soc 2014; 11(9): 1454-1465.

27. Nieves Ortega R, Rosin C, Bingisser R, Nickel CH. Clinical scores and formal triage for screening of sepsis and adverse outcomes on arrival in an emergency department all-comer cohort. J Emerg Med 2019; 57(4): 453-460.e2. 